\title{
Molecular Mechanism for the Regulation of Microcystin Toxicity to Protein Phosphatase 1 by Glutathione Conjugation Pathway
}

\author{
Wansong Zong, ${ }^{1}$ Xiaoning Wang, ${ }^{1}$ Yonggang Du, ${ }^{1}$ Shuhan Zhang, \\ Ying Zhang, ${ }^{1}$ and Yue Teng ${ }^{2}$ \\ ${ }^{1}$ College of Geography and Environment, Shandong Normal University, 88 East Wenhua Road, Jinan, Shandong 250014, China \\ ${ }^{2}$ School of Environmental and Civil Engineering, Jiangnan University, 1800 Lihu Avenue, Wuxi, Jiangsu 214122, China
}

Correspondence should be addressed to Wansong Zong; wansongzong@hotmail.com

Received 18 August 2016; Revised 8 November 2016; Accepted 15 November 2016; Published 27 February 2017

Academic Editor: Stefano Curcio

Copyright (C) 2017 Wansong Zong et al. This is an open access article distributed under the Creative Commons Attribution License, which permits unrestricted use, distribution, and reproduction in any medium, provided the original work is properly cited.

Glutathione (GSH) conjugation was an important pathway to regulate the toxicity of microcystins (MCs) targeted to protein phosphatases. To explore the specific molecular mechanism for GSH detoxification, two typical MC-GSHs (derived from MCLR and MCRR) were synthesized, prepared, and purified according to previous research. Then, the reduced inhibition effect for MCGSHs on protein phosphatase 1 was verified by comparing with their original toxins. To further clarify the molecular mechanism for MC-GSHs detoxification, we evaluated the interactions between MCs/MC-GSHs and PP1 with the assistance of MOE molecule simulation. When GSH was introduced to MCs, the covalent binding ( $\mathrm{Mdha}^{7}$ to $\mathrm{Cys}_{273}$ ), the hydrophobic interaction (Adda ${ }^{5}$ with PP1), the hydrogen bonds (especially for $\mathrm{Lys}^{2}-\mathrm{Arg}_{96}$ and $\mathrm{Glu}^{6}-\mathrm{Tyr}_{272}$ ), the covalent combination (between Mdha ${ }^{7}$ and $\mathrm{Cys}_{273}$ ), and the ion bonds (between $\mathrm{Mn}^{2+}$ and $\mathrm{Asn}_{124} / \mathrm{His}_{248} / \mathrm{Asp}_{64} / \mathrm{His}_{66}$ ) of MCLR/MCRR-PP1 complexes weakened to a certain extent, while the ion bonds between $\mathrm{Mn}^{2+}$ and $\mathrm{His}_{173} / \mathrm{Asp}_{92}$ residues increased. It was not difficult to find that the toxicity of MCs was closely related to the above sites/interactions and the above key information for MCs-PP1; MC-GSHs-PP1 complexes were important for clarifying the detoxification mechanism of MC-GSHs pathway. This study offers a comprehensive cognition on MCs toxicity regulation and provides valid theoretical support to control their potential risk.

\section{Introduction}

Microcystins (MCs) pose a worldwide health threat to humans and animals due to their increasing presence in aquatic environments as well as in water distribution systems $[1,2]$. MCs are a class of hepatic heptapeptides produced by toxic cyanobacteria and posed a risk to environment when released as metabolic byproducts or during cyanobacteria cell lysis [3]. MCs shared the common structure of cyclo(-D-Ala ${ }^{1}-\mathrm{L}-\mathrm{X}^{2}$-D-isoAsp ${ }^{3}-\mathrm{L}-\mathrm{Z}^{4}-\mathrm{Adda}^{5}$-DisoGlu ${ }^{6}-\mathrm{N}$-methyldehydro-Ala ${ }^{7}$ ), in which $\mathrm{X}^{2}$ and $\mathrm{Z}^{4}$ were two variable amino acids, Adda $^{5}$ was 3-amino-9-methoxy2,6,8-trimethyl-10-phenyldeca-4,6-dienoic acid [4]. Due to the two variable amino acids and methylation/demethylation of other residues, there are more than 80 variants [5]. Among these toxins, MCLR and MCRR ( $\mathrm{L}$ and R stand for variable amino acids Leu and Arg, resp.) are the most frequently found and studied variants $[6,7]$.
Toxicology experiments showed MCs had selectively hepatotoxicity through specific inhibition of protein phosphatases 1 (PP1) and 2A (PP2A), which in turn induced the hyperphosphorylation of some key control proteins in signal transduction [8]. The imbalance of protein phosphorylation/dephosphorylation promotes the oxidative damage of proteins and DNA, leading to cell structure disruption, apoptosis, liver necrosis, and intrahepatic hemorrhage [4, $7,9]$. In hepatic cells, MCs undergo a two-step interaction with PPs: the first step involves reversible binding that leads to rapid inhibition of catalytic activity; the second step involves formation of a covalent bond between the $\mathrm{N}$ methyldehydroalanine residue $\left(\mathrm{Mdha}^{7}\right)$ and a nucleophilic site on the PPs, leading to irreversible inactivation [10]. Crystal structure analysis of MCs-PP1/PP2A complexes confirms that MCs mainly attack the active site pocket of PP1/PP2A catalytic subunits through hydrogen bonds and ion bonds, and the hydrophobic cage structure adjacent to the active site 
pocket just can accept the hydrophobic side-chain of $\mathrm{Adda}^{5}$ [11].

The above features of MC-PP1/PP2A complexes may determine the typical inhibition effect of MCs on PP1/PP2A. Blocking or destroying their combination is important to regulate the inhibition effect of MCs on PP1/PP2A. Recent studies on MC regulation showed there is an enzymatic pathway for MCs detoxification via glutathione S-transferases (GSTs) [12-15]. The GST-derived metabolites of MCs are glutathione conjugates (MC-GSHs), which are obtained through the nucleophilic reaction of GSH thiol to the unsaturated carbonyl in $\mathrm{Mdha}^{7}$ of MCs $[14,16]$. GSH conjugation appears to be the key step for MCs detoxication as MC-GSHs had lower toxicity and higher hydrophily compared with original toxins [17]. To date, the preparation and quantitative methods for MC-GSHs have been proposed, and the detoxification effect of GSH (with the aid of PPs inhibitory assays) has been widely studied. However, limited information on the structural features of MC-GSH-PPs complexes restricts the research on the interactions between MC-GSHs and PP1/PP2A. For this reason, the specific molecular mechanism for MC-GSH detoxification is not yet very clear. Thus, clarifying the molecular mechanism for the regulation of MCs toxicity (targeted to PPs) by MC-GSH pathway is of great importance and urgency.

To explore the detoxification mechanism of GSH to MCs toxicity, two primary MC-GSHs originated from MCLR and MCRR were synthesized through electrophilic addition reaction. After chromatography preparation and purification, their biological toxicity target to PP1 was evaluated and compared with that of MCLR and MCRR. To clarify the molecular mechanism for MC-GSH detoxification, we further evaluated the interactions between MCs/MC-GSHs and PP1 with the assistance of Molecular Operating Environment (MOE) software molecule simulation. MOE is an interactive, windowsbased chemical computing and molecular modeling tool and can simulate the interaction between toxicant and protein. On the basis of toxicity evaluation and molecular simulation, the key action sites and interaction modes for the toxicity of MCLR/MCRR and MCLR-GSH/MCRR-GSH were identified and compared. Accordingly, the specific molecular mechanism for MCs toxicity regulation (by MC-GSHs pathway) was clarified.

\section{Materials and Methods}

2.1. Materials. MCLR and MCRR standards were purchased from Sigma (Saint-Quentin Fallavier, France). PP1 (1500 U/ $\mathrm{mL}$ ) from rabbit skeletal muscle were obtained from EMD Millipore (Darmstadt, Germany). HPLC grade acetonitrile, trifluoroacetic acid, and methanol were obtained from Merck (Darmstadt, Germany). Bovine serum albumin, dithiothreitol, GSH, $\mathrm{MnCl}_{2}$, p-nitrophenyl disodium orthophosphate, and tris(hydroxymethyl)aminomethane were purchased from Sinopharm (Shanghai, China).

2.2. Addition Reaction of GSH to MCs. In order to prepare MC-GSHs, $2 \mu \mathrm{M}$ MCLR/MCRR and $500 \mu \mathrm{M}$ GSH were mixed in $5 \% \mathrm{~K}_{2} \mathrm{CO}_{3}$ and incubated for $2 \mathrm{~h}$ at room temperature [18]. Then, the reaction mixtures were neutralized with $0.2 \mathrm{M} \mathrm{HCl}$ and applied to conditioned Cleanert $\mathrm{C}_{18}$ SPE cartridges (500 mg, Bonna-Agela) that were rinsed with $10 \mathrm{~mL}$ methanol and $15 \mathrm{~mL}$ water. The impurities were eluted with $10 \mathrm{~mL} 10 \%$ methanol and MCs/MC-GSHs were eluted with $10 \mathrm{~mL} 80 \%$ methanol. The eluted samples were evaporated to dryness in $\mathrm{N}_{2}$ flow and resuspended in $1 \mathrm{~mL}$ acetonitrile. The samples were stored in $-20^{\circ} \mathrm{C}$ before HPLC and mass spectra (MS) analysis.

\subsection{MCs and MC-GSHs Analysis}

2.3.1. Directed MS Analysis of MCs and MC-GSHs. The crude extracts for MCs and MC-GSHs were analyzed by a maXis UHR-TOF mass spectrometer (Bruker Daltonics). Samples were mixed with isometric acetonitrile (containing 0.1\% trifluoroacetic acid) and injected into MS spectrometer with a syringe pump at $5 \mu \mathrm{L} / \mathrm{min}$. MS parameters were set as follows: positive ion mode, electrospray source voltage $4.2 \mathrm{kV}$, cone voltage $0.5 \mathrm{kV}$, desolvation gas $\mathrm{N}_{2} 0.5$ bar, dry gas $\mathrm{N}_{2} 4 \mathrm{~L} / \mathrm{min}$, dry gas heater $180^{\circ} \mathrm{C}$, and scan range $400-1500$. Data acquisition was controlled with the Compass software and MCs/MCGSHs could be detected according to their $\mathrm{m} / z$ signals.

2.3.2. MS/MS Analysis of MCs and MC-GSHs. MC-GSHs were further identified by comparing their specific secondary ions with those of MCs standards. MC-GSHs were collected from LC separation at their specific retention times and injected into MS spectrometer with a syringe pump at $5 \mu \mathrm{L} / \mathrm{min}$. MS/MS parameters were set as Section 2.3.1 except that $\mathrm{N}_{2}$ collision gas was used and collision energies were adjusted at $50 \mathrm{eV}$.

2.4. MC-GSHs Preparation. Obtaining purified MC-GSHs was the precondition for biological toxicity evaluation. For this reason, resuspended samples containing MCs and MCGSHs were further separated using a Great Eur-Asia $\mathrm{C}_{18}$ column $(9.4 \times 250 \mathrm{~mm}, 5 \mu \mathrm{m}, 120 \AA)$ on the previously mentioned HPLC-MS system. Instrument parameters were set as in Section 2.3 except that the injection volume was $100 \mu \mathrm{L}$ and the elution rate was $2 \mathrm{~mL} / \mathrm{min}$. Subsequently, the purified MC-GSHs were collected manually according to their specific retention times, evaporated to dryness with $\mathrm{N}_{2}$, and dissolved in $200 \mu \mathrm{L}$ methanol. MS analysis of isolated MC-GSHs was performed to evaluate their concentrations and purity with MCLR/MCRR standards as references.

2.5. Protein Phosphatase Inhibition Assay for MCs and MCGSHs. The biological toxicity of MCs and MC-GSHs was evaluated by a colorimetric protein phosphatase inhibition assay $[19,20]$. Firstly, PP1 was diluted to $5 \mathrm{U} / \mathrm{mL}$ with a freshly prepared buffer of $50 \mathrm{mM}$ tris(hydroxymethyl)aminomethane- $\mathrm{HCl}$ ( $\mathrm{pH}$ 7.4), $2 \mathrm{mM}$ dithiothreitol, $1 \mathrm{mM} \mathrm{MnCl}_{2}$, and $1 \mathrm{~g} / \mathrm{L}$ bovine serum albumin. Then, $10 \mu \mathrm{L}$ PP1 was added to $100 \mu \mathrm{L}$ test samples in a 96 -well polystyrene microplate. With gentle shaking, the microplate was kept at $25.0^{\circ} \mathrm{C}$ for a quarter-hour and p-nitrophenyl disodium orthophosphate was added. After $1 \mathrm{~h}$, the absorbances of incubated samples ( $p$-nitrophenol production) were measured in 


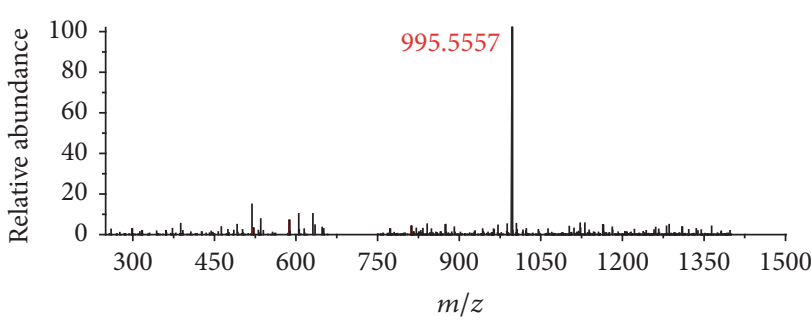

(a)

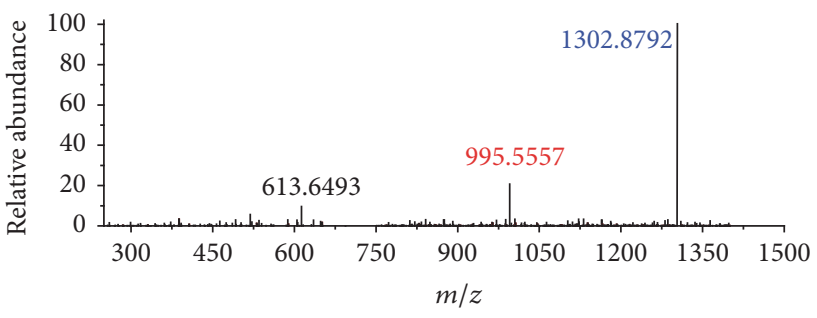

(c)

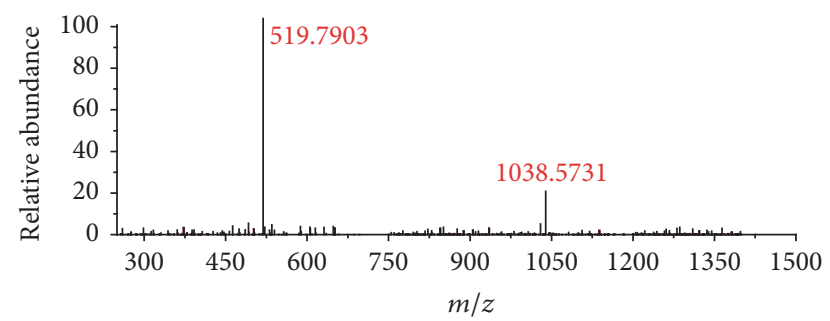

(b)

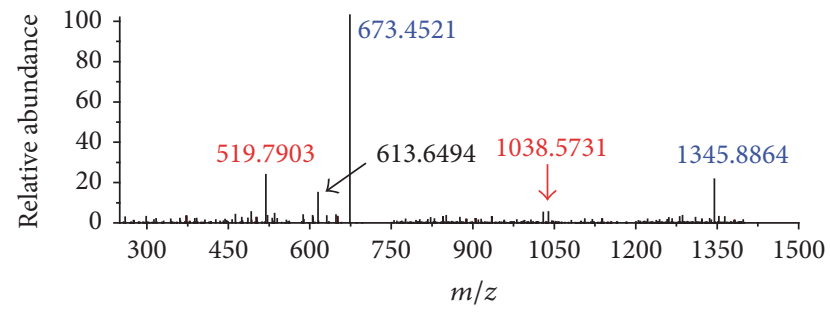

(d)

FIGURE 1: MS analysis for MCLR (a), MCRR (b), and the GSH electrophilic addition samples to prepare MCLR-GSH (c) and MCRR-GSH (d).

a THERMO/max microplate reader. The inhibition of test samples on PP1 could be expressed as follows:

$$
I_{\mathrm{PP} 1}=\frac{\left(A_{\text {control }}-A_{\text {sample }}\right)}{A_{\text {control }}} \times 100 \% \text {, }
$$

where $A_{\text {control }}$ and $A_{\text {sample }}$ were the absorbances of reference sample (without PP1) and test sample at $405 \mathrm{~nm}$, respectively.

2.6. Molecular Simulation for the Interaction between PP1 and MCs/MC-GSHs. Molecular simulation calculations were performed with MOE software (version number 14.09). The original structure for MCLR-PP1 complex was obtained from Protein Data Bank (PDB code 1FJM, http://www.rcsb.org/ pdb/home/home.do). Models for MCLR and PP1 were extracted based on the structure of MCLR-PP1. Models for MCRR, MCLR-GSH, and MCRR-GSH were prepared based on the structure of MCLR. Before calculations, receptor PP1 was protonated by adding hydrogen atoms and small molecule ligands were minimized for energy optimization. Then, the interactions between toxins and PP1 were simulated (Amber 10: EHT, Solvation: R-Field) and the key parameters such as the total energies, total combination areas, hydrogen bonds, and ionic bonds for main interaction sites were obtained for clarifying the detoxification mechanism of MCGSHs pathway. To keep the consistency of experiment conditions with PP1 inhibition assay, the experiment conditions for MOE simulation were set as follows: reaction temperature $25.0^{\circ} \mathrm{C}, \mathrm{pH} 7.4$, and salt $0.05 \mathrm{M}$.

\section{Results and Discussion}

3.1. MC-GSHs Synthesis and Identification. With conjugation with GSH, MCs might transform into specific MC-GSHs with different molecular weights which could be probed by mass spectrograph. For MCLR with a molecular weight of 994.5482, its primary MS signal was detected at $m / z$ 995.5558 (Figure 1(a)), corresponding to the single-proton product of native toxin. For MCRR with a molecular weight of 1037.5652, two primary MS signals were detected at $\mathrm{m} / \mathrm{z}$ 519.7903 and 1038.5731 (Figure 1(b)), corresponding to the double-proton and single-proton products. After electrophilic addition samples, MCLR and MCRR still exist (Figures 1(c) and 1(d)). However, they had lower intensities than the newly formed ions with MS signals at $\mathrm{m} / z 1302.8792$ and $673.4521 / 1345.8864$. As GSH was about $307.3235 \mathrm{Da}$, the above MS signals should be attributed to the addition products of GSH to MCLR or MCRR [21]. In addition, a product with MS signal at $m / z 613.6493$ was also found in both addition samples. This product should be attributed to the directed oxidation of sulfhydryl groups in two GSH, forming oxidized GSH (GSSG).

Molecular weight change could not provide further assistance for the identification of MC-GSHs. Accordingly, the specific generative mechanism of MC-GSHs was confirmed by comparing their secondary structures with MCLR and MCRR (with the assistance of Compass Isotope Pattern software). MS/MS analysis showed partial CID fragments of MCLR $(m / z$ 995.5558) were detected at $\mathrm{m} / z$ 213.0831, 286.1477, 553.3069, 682.3956, and 866.5147 (Figure 2(a)), corresponding to the secondary structures of [GluMdha $+\mathrm{H}]^{+},[\text {MeAsp-Arg }+\mathrm{H}]^{+},[$Mdha-Ala-Leu-MeAsp-Arg $+\mathrm{H}]^{+}, \quad$ [Arg-Adda-Glu-Mdha $\left.+\mathrm{H}\right]^{+}$, and [Mdha-Ala-LeuMeAsp-Arg-Adda $+\mathrm{H}]^{+} /[\text {Arg-Adda-Glu-Mdha-Ala-Leu+H }]^{+}$ $[22,23]$. For MCRR $(m / z 519.7903, z=2)$, its primary CID fragments were detected at $m / z$ 213.0831, 286.1477, 298.6720, 413.7556, 440.2252, 455.2741, 484.2768, and 599.3552 (Figure 2(b)), corresponding to the ions of [Glu$\mathrm{Mdha}+\mathrm{H}]^{+},[\mathrm{MeAsp}-\text { Arg }+\mathrm{H}]^{+},[$Mdha-Ala-Arg-MeAsp-Arg $+2 \mathrm{H}]^{2+}, \quad$ [Ala-Arg-MeAsp-Arg-Adda $\left.+2 \mathrm{H}\right]^{2+}, \quad[$ Mdha-AlaArg-MeAsp $+\mathrm{H}]^{+}, \quad[\text { Arg-Adda-Glu-Mdha-Ala-Arg }+2 \mathrm{H}]^{2+}$, 


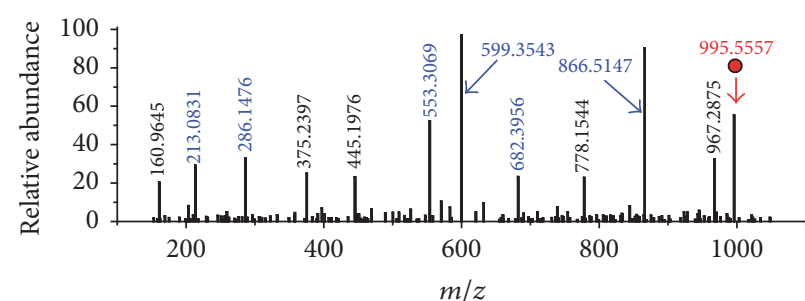

(a)

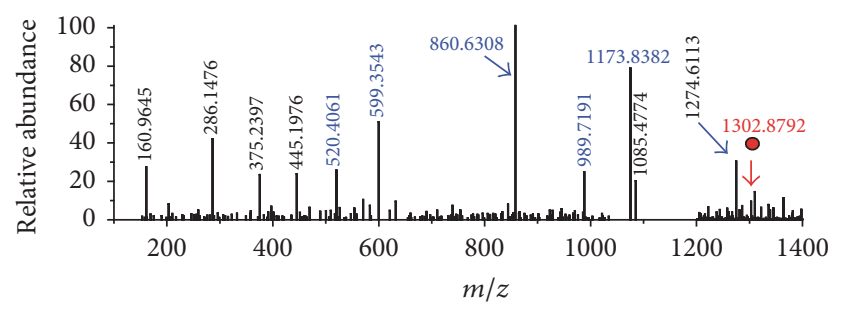

(c)

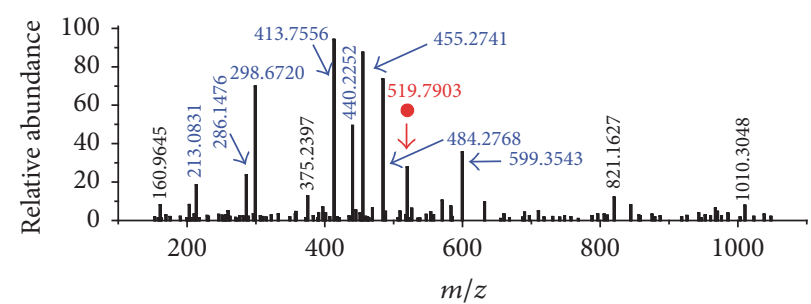

(b)

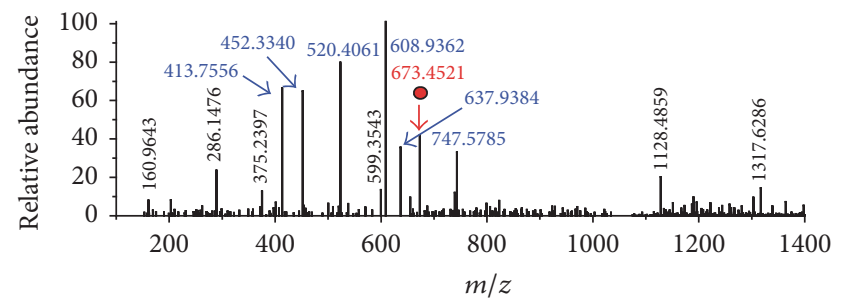

(d)

FIGURE 2: MS/MS analysis of MCLR (a), MCRR (b), and the identified electrophilic addition products MCLR-GSH (c) and MCRR-GSH (d). Conditions: $m / z$ signals at $995.5557,519.7903,1302.8792$, and 673.4521 correspond to the precursor ions of MCLR, MCRR, MCLR-GSH, and MCRR-GSH, respectively.

[Arg-MeAsp-Arg-Adda-Glu-Mdha+2H $]^{2+}$, and [Arg-Adda$\mathrm{Glu}+\mathrm{H}]^{+} /[\text {MeAsp-Arg-Adda }+\mathrm{H}]^{+}$.

Based on the same strategy, the CID fragments of MCLR-GSH and MCRR-GSH could also be obtained. For MCLR-GSH with the $m / z$ at 1302.8792 (Figure 2(c)), it had several identical fragment ions as that of MCLR (e.g., 160.9654 and 286.1477). In addition, MCLR-GSH also had partial new CID fragments at $m / z 520.4061,860.6308,989$ .7195 , and 1173.8082 , corresponding to the ions of [Glu$\mathrm{Mdha}+\mathrm{H}]^{+}+307.3230, \quad$ [Mdha-Ala-Leu-MeAsp-Arg $\left.+\mathrm{H}\right]^{+}+$ 307.3239, [Arg-Adda-Glu-Mdha $+\mathrm{H}]^{+}+307.3239$, and [MdhaAla-Leu-MeAsp-Arg-Adda $+\mathrm{H}]^{+} /[$Arg-Adda-Glu-Mdha-Ala$\mathrm{Leu}+\mathrm{H}]^{+}+307.3235$. It was not difficult to find that MCLRGSH fragments containing $\mathrm{Mdha}^{7}$ were sustained by a $307.3235 \pm 0.0005 \mathrm{Da}$ difference with MCLR. In accordance with data in literature [24], these products should be from the additive reaction of GSH to the $\mathrm{C}=\mathrm{C}$ bond in $\mathrm{Mdha}^{7}$ residual. MCRR-GSH (Figure 2(d)) $(\mathrm{m} / \mathrm{z}=$ 673.4521) also had several identical fragments as that of MCRR except for [Glu-Mdha $+\mathrm{H}]^{+}+307.3230$, [Mdha-AlaArg-MeAsp-Arg+2H] ${ }^{2+}+153.6619$, [Mdha-Ala-Arg-MeAsp+ $\mathrm{H}]^{+}+307.3233, \quad$ [Arg-Adda-Glu-Mdha-Ala-Arg $\left.+2 \mathrm{H}\right]^{2+}+$ 153.6621, and [Arg-MeAsp-Arg-Adda-Glu-Mdha $+2 \mathrm{H}]^{2+}+$ 153.6616. Undoubtedly, MCRR-GSH fragments with $\mathrm{Mdha}^{7}$ residual were sustained by a $307.3235 \pm 0.0005 \mathrm{Da}$ difference with MCRR. According to the above analysis, GSH was undoubtedly added to the Mdha $\mathrm{C}=\mathrm{C}$ bond of MCRR and formed MCRR-GSH.

\subsection{Biological Toxicity Evaluation of MCs and MC-GSHs} Target to PP1. To evaluate and compare the potential toxicity of MCLR, MCRR, and MC-GSHs to PP1, related MCGSHs were prepared and purified with SPE and preparative chromatography techniques. The preparation and purification information for MC-GSHs were listed in Table 1.
TABLE 1: Preparation and purification information for MCLR-GSH and MCRR-GSH.

\begin{tabular}{lcccc}
\hline $\begin{array}{l}\text { Conjugation } \\
\text { products }\end{array}$ & Eluted time & Concentration & Total volume & Purity \\
\hline MCLR-GSH & $12.54 \mathrm{~min}$ & $\approx 1285 \mu \mathrm{mol} / \mathrm{L}^{\mathrm{b}}$ & $10 * 100 \mu \mathrm{L}$ & $98.3 \%$ \\
MCRR-GSH & $8.43 \mathrm{~min}$ & $\approx 1094 \mu \mathrm{mol} / \mathrm{L}$ & $10 * 100 \mu \mathrm{L}$ & $98.7 \%$ \\
\hline
\end{tabular}

a: collection time was set for $0.5 \mathrm{~min}( \pm 0.25 \mathrm{~min}$ around the eluted time). b: with $200 \mu \mathrm{mol} / \mathrm{L}$ MCLR (MCRR) serving as the inner standard for quantification and assuming MCLR and MCLR-GSH (MCRR and MCRRGSH) had approximate protonated efficiencies.

c: purity was directed calculated by MS signals and defined as MC-GSH/(MC + MC-GSH) $* 100 \%$

As MC-GSHs had higher concentrations (ranging from $1094 \mu \mathrm{g} / \mathrm{L}$ to $1285 \mu \mathrm{g} / \mathrm{L}$ ) and higher purity (>98.3\%), the prepared samples could be directed used to evaluated the toxicity of MC-GSHs.

Based on PP1 inhibition experiment, the inhibition curves for MCLR, MCRR, and their conjugation products were plotted and their $\mathrm{IC}_{50}$ was calculated out. Figure 3 showed MC-GSHs had lower toxicity than their native toxins in the sequence of MCLR $\left(\mathrm{IC}_{50}=2.5 \mu \mathrm{g} / \mathrm{L}\right)>\mathrm{MCRR}\left(\mathrm{IC}_{50}\right.$ $=24.4 \mu \mathrm{g} / \mathrm{L})>$ MCLR-GSH $\left(\mathrm{IC}_{50}=86.6 \mu \mathrm{g} / \mathrm{L}\right)>$ MCRRGSH $\left(\mathrm{IC}_{50}=98.7 \mu \mathrm{g} / \mathrm{L}\right)$. Similar to previous studies $[17,21]$, it was not difficult to find that GSH conjugation was an effective way to control the toxicity of MCs. Though the toxicity of MC-GSHs was obviously decreased, the secondary biotoxicity of MC-GSHs was real and nonnegligible. As a result, the secondary pollution of MC-GSHs also deserved further attention.

3.3. Molecular Mechanism for the Different Toxicity of $M C s$ and MC-GSHS on PP1. Although toxicity experiment revealed GSH conjunction had obvious regulation effect on 


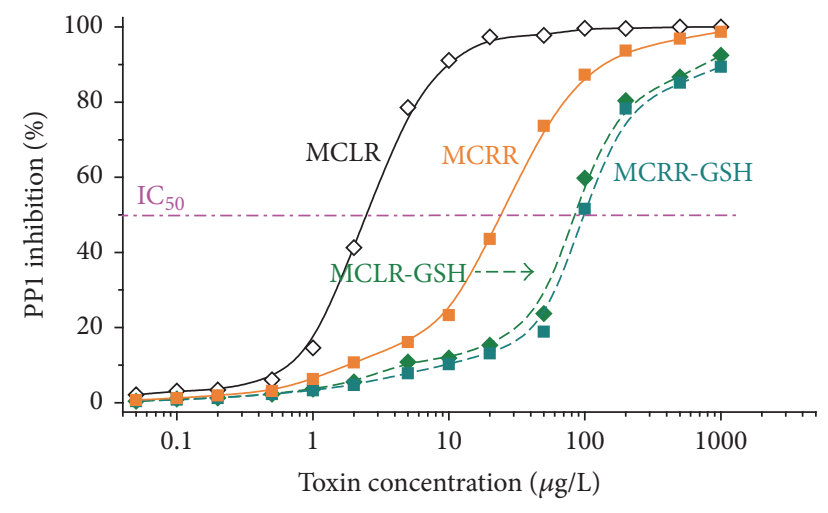

FIGURE 3: Inhibition curves for MCLR, MCRR, and related MCLRDBPs on PP1. IC I0 $_{50}$ for MCLR, MCRR, MCLR-GSH, and MCRRGSH on PP1 were about $2.5 \pm 0.2 \mu \mathrm{mol} / \mathrm{L}, 24.4 \pm 0.5 \mu \mathrm{mol} / \mathrm{L}, 86.6 \pm$ $1.2 \mu \mathrm{mol} / \mathrm{L}$, and $98.7 \pm 1.0 \mu \mathrm{mol} / \mathrm{L}$, respectively.

MC toxicity, the detoxification mechanism has not been clarified as limited information was available on the structural features of MC-GSH-PP1 complexes. For these reasons, the specific interaction between MCLR, MCRR, MC-GSHs, and PP1 should be further explored with the assistance of molecular simulation. Between MCs and PP1, there were a reversible binding step through hydrogen bonding, ion bonding, hydrophobic interaction involving $\mathrm{Adda}^{5}$ residue, and an irreversible covalent bonding step involving $\mathrm{Mdha}^{7}$ residue and a nucleophilic site (with MCLR-PP1 complex serving as an example, Figure 4) $[15,25]$. Accordingly, the total energies and total combination areas of toxin-PP1 complexes, the combination areas of $\mathrm{Adda}^{5}$ and $\mathrm{Mdha}^{7}$ residuals to PP1, the hydrogen bonds, and ionic bonds for main interaction sites were selected as the key parameters to assess the detoxification mechanism of GSH conjunction.

Figure 5 showed the simulation information for the combination area and energy changes of toxin-PP1 complexes. Compared to the reversible binding step, the irreversible binding of MCLR/MCRR to PP1 both increased in total combination areas (Figure 5(a)), indicating that covalent binding of $\mathrm{Mdha}^{7}$ to $\mathrm{Cys}_{273}$ promoted the interactions between MCLR/MCRR and PP1. Though the specific covalent binding was destroyed by the introduction of GSH, the total combination areas still showed marked increasing tendency. In fact, the toxicity of MC-GSHs was much lower than their original toxins; the increments of combination areas should be attributed to the direct combination of GSH residue to PP1. The interpretation could be verified by the increased combination area for $\mathrm{Mdha}^{7} / \mathrm{Mdha}^{7}-\mathrm{GSH}$ with PP1: the combination areas increased after irreversible combination and significantly increased when GSH was introduced to MCs (Figure 5(b)). If the combination areas for $\mathrm{Mdha}^{7} / \mathrm{Mdha}^{7}$ GSH with PP1 were subtracted, the combination areas represented a marked slowdown. Accordingly, it could be ascertained that $\mathrm{Mdha}^{7}$ residue has certain relevance with MC toxicity. The toxicity of MCs to PP1 could be reduced by blocking the covalent binding of Mdha ${ }^{7}$ to PP1 by GSH conjunction pathway.
For Adda residue involved in hydrophobic interaction (Figure 5(c)), the combination areas for irreversible binding step were increased compared to reversible binding step. When GSH was introduced, the combination areas for $\mathrm{Adda}^{5}$ with PP1 represented marked slowdown. The combination areas for Adda ${ }^{5}$ with PP1 showed a positive correlation with the toxicity of MCs and their GSH conjunction products. Hydrophobic interaction for $\mathrm{Adda}^{5}$ with PP1 was an important factor for the toxicity of MCs and MC-GSHs. The toxicity of MCs to PP1 could also be reduced by blocking the hydrophobic interaction of $\mathrm{Adda}^{5}$ to PP1.

Compared to the reversible binding step, the irreversible binding of MCs to PP1 was more stable due to the significant decline in total energies (Figure 5(d)). Though GSH conjunction blocked up the combination of $\mathrm{Mdha}^{7}$ to $\mathrm{Cys}_{273}$ residue, the total energies for these complexes had more apparently downtrend. Actually, the conjunction products had much lower toxicity than original toxins and there were no direct relations between total energy changes and toxicity. The setback values for combination energies should be attributed to the extra interaction between GSH residues and PP1 as the increments of combination areas between MC-GSHs and PP1 weakened the surface energy of PP1.

Figure 6 showed the simulation information for hydrogen bonds and covalent bonds of toxin-PP1 complexes. Compared to the reversible binding step, the total hydrogen bonds (MCs/MC-GSHs with PP1 and $\mathrm{H}_{2} \mathrm{O}$ ) for the irreversible binding step of MC-PP1 complexes were obviously promoted (Figure 6(a)). When GSH was introduced, the combination of toxins to PP1 was blocked up and the hydrogen bonds for toxin-PP1 complexes were dramatically decreased. The changing trends for total hydrogen bonds also could be applied to the hydrogen-bond change for toxin residues with $\mathrm{PP} 1$ or $\mathrm{H}_{2} \mathrm{O}$. The positive correlation between hydrogen bonds and toxin toxicity showed hydrogen bonds also were important factors for the toxicity of MCs and MC-GSHs. As MCs and MC-GSHs had multiple potential hydrogen bonding sites to PP1, the hydrogen bond for single interaction site was obtained and illustrated in Figure 6(b). After irreversible binding, the hydrogen bonds for interaction sites Lys ${ }^{2}$ with $\mathrm{Arg}_{96}$ and $\mathrm{Glu}^{6}$ with $\mathrm{Tyr}_{272}$ were promoted, the hydrogen bonds for IsoAsp ${ }^{3}$ with $\mathrm{Arg}_{96}$ were decreased in some degree, and the hydrogen bonds for other interaction sites showed no consistent trends. Accordingly, the hydrogen bonds for interaction sites $\mathrm{Lys}^{2}-\mathrm{Arg}_{96}$ and $\mathrm{Glu}^{6}-\mathrm{Tyr}_{272}$ were closely related to the toxicity of MCs and MC-GSHs. Although three new types of hydrogen bonds for interaction sites Adda ${ }^{5}$ with $\operatorname{Arg}_{221}$, Mdha ${ }^{7}$-GSH with $\mathrm{Asn}_{278}$, and Mdha ${ }^{7}$-GSH with $\mathrm{Asn}_{271}$ were formed after GSH conjugation, the hydrogen bonds for interaction sites $\mathrm{Arg}^{4}$ with $\mathrm{Glu}_{275}$, $\mathrm{Lys}^{2}$ with $\mathrm{Arg}_{96}$, $\mathrm{Glu}^{6}$ with $\mathrm{Tyr}_{272}$, and Mdha ${ }^{7}$ with $\mathrm{Gly}_{274}$ still represented marked slowdown. Accordingly, the regulation effect of GSH for MCs toxicity was closely related to the above interaction sites. The toxicity of MCs to PP1 could be controlled by enhancing the hydrogen bonds for interaction sites $\mathrm{Lys}^{2}$ with $\mathrm{Arg}_{96}$ and $\mathrm{Glu}^{6}$ with $\mathrm{Tyr}_{272}$ and by blocking the hydrogen bonds for interaction sites IsoAsp ${ }^{3}$ with $\mathrm{Arg}_{96}, \mathrm{Arg}^{4}$ with $\mathrm{Glu}_{275}$, $\mathrm{Lys}^{2}$ with $\mathrm{Arg}_{96}$, $\mathrm{Glu}^{6}$ with $\mathrm{Tyr}_{272}$, and Mdha ${ }^{7}$ with $\mathrm{Gly}_{274}$. 


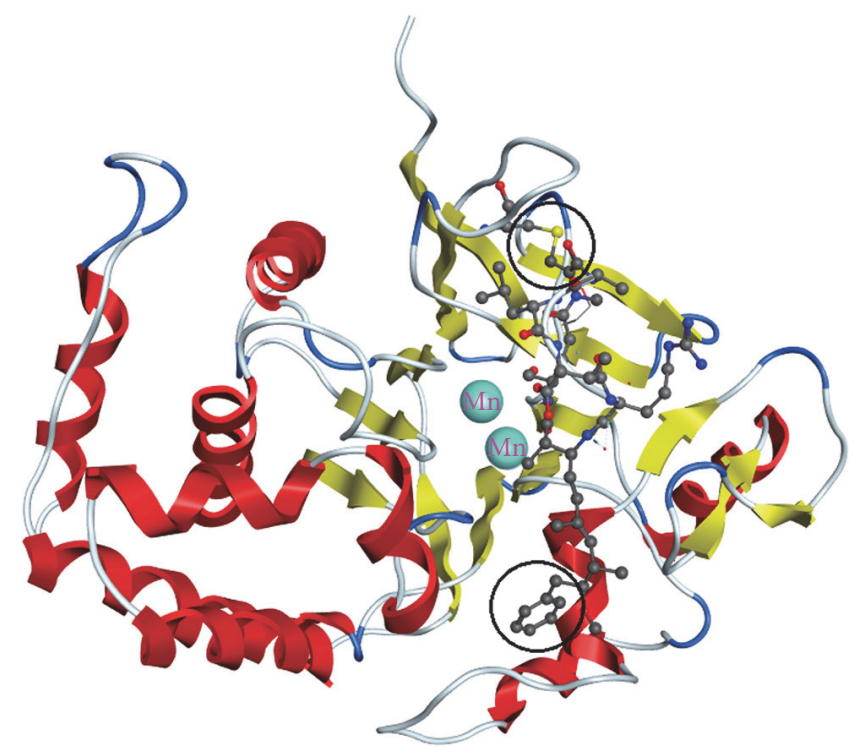

(a)

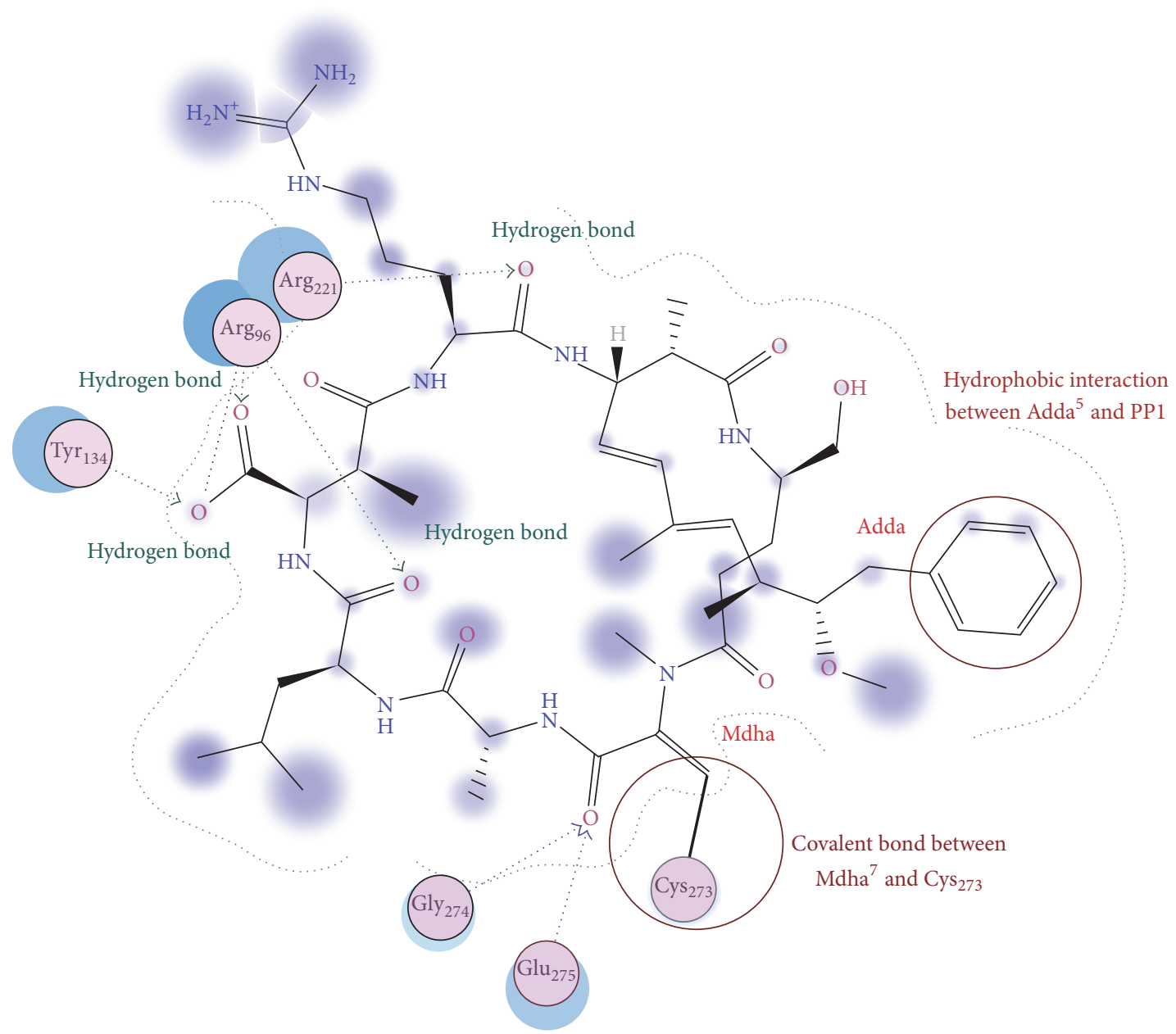

(b)

FIGURE 4: Molecular simulation results of MCLR and PP1 system. (a) The stereoscopic structure of MCLR-PP1 complex displayed in cartoon form. (b) The interaction between MCLR and related amino acid residues in PP1. 


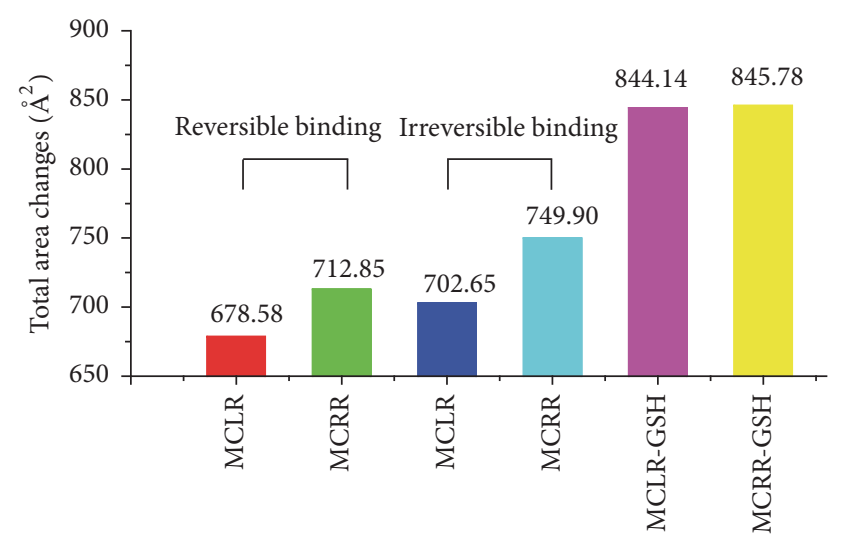

(a)

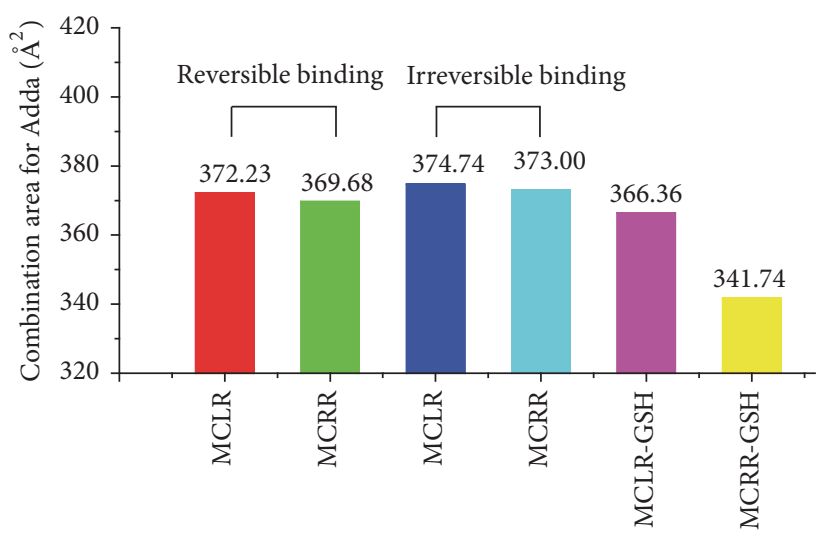

(c)

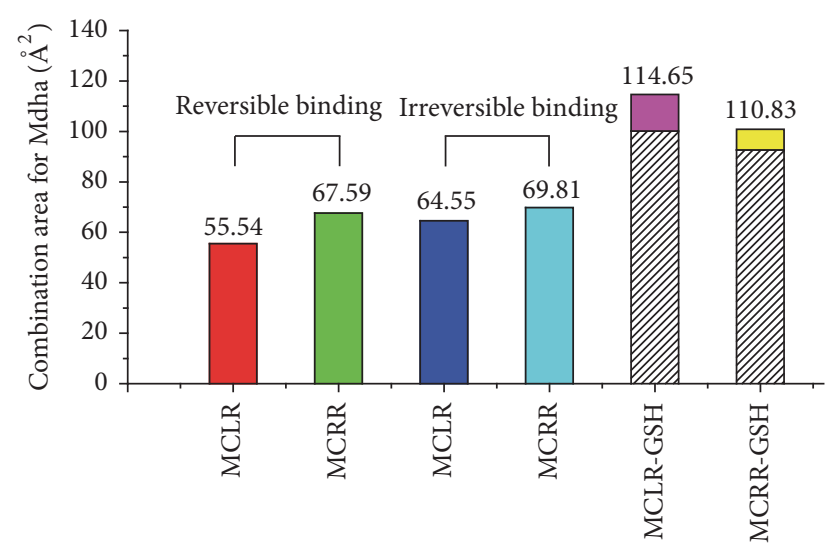

पIIIA Area for GSH residual

(b)

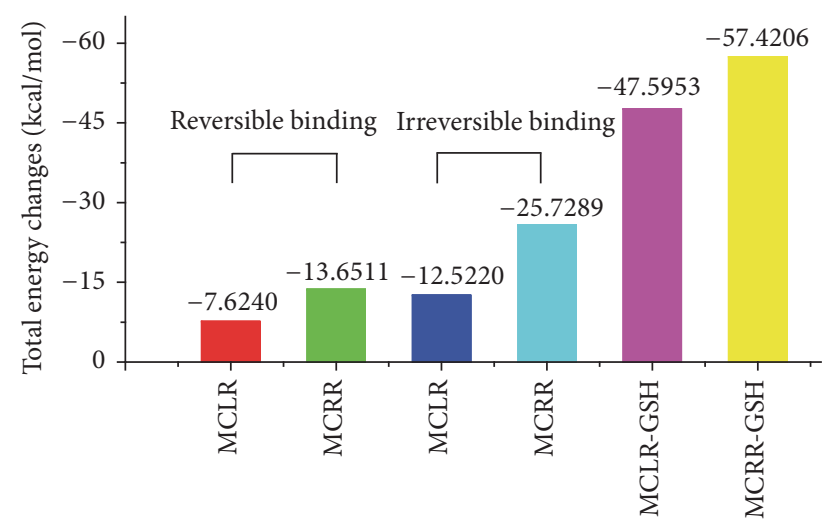

(d)

FIGURE 5: Molecular simulation results for the total combination area changes (a), the combination area changes for Mdha (b) and Adda (c) residuals, and the total energy changes $(\mathrm{d})$ of target complexes.

As the irreversible binding step between MCs and PP1 involved the nucleophilic site $\mathrm{Cys}_{273}$, data for the interactions of toxins with residue $\mathrm{Cys}_{273}$ were also obtained (Figure 6(c)). For the reversible binding step of MCs to PP1, Cys ${ }_{273}$ had no direct interaction with Mdha but combined with $\mathrm{Asn}_{278}$ residue by hydrogen bonds. For the irreversible binding step, the formation of covalent bonds between $\mathrm{Cys}_{273}$ and Mdha ${ }^{7}$ should overcome the hydrogen bonds between $\mathrm{Cys}_{273}$ and $\mathrm{Asn}_{278}$. When GSH was introduced, the interactions between $\mathrm{Cys}_{273}$ and Mdha/Asn ${ }_{278}$ were destroyed. Considering the inhibition effect of MCs and MC-GSHs on PP1, the covalent combination of Mdha residue and $\mathrm{Cys}_{273}$ had certain relevance with the toxicity of MCs and MC derivatives.

As PP1 was a type of metalloenzyme and regulated by two $\mathrm{Mn}^{2+}$ ions, the discrepant inhibition effect of MCs and MC-GSHs on PP1 might be mediated by $\mathrm{Mn}^{2+}$ ions. Specific interactions involving $\mathrm{Mn}^{2+}$ ions in PP1 catalytic center were also investigated. For the first $\mathrm{Mn}^{2+}$ ion (Figure 7(a)), the total ion bonds were promoted with irreversible binding of MCs with PP1 (mainly attributed to the new ion bond between $\mathrm{Mn}^{2+}$ and $\left.\mathrm{Asn}_{124}\right)$. When GSH was introduced, the ion bond between $\mathrm{Mn}^{2+}$ and $\mathrm{His}_{248}$ was totally destroyed. However, due to the significantly increased ion bond between $\mathrm{Mn}^{2+}$ and $\mathrm{His}_{173}$, the ion bonds for MC-GSH-PP1 complexes are still enhanced to a certain extent. Accordingly, the interaction for $\mathrm{Mn}^{2+}$ and $\mathrm{His}_{173}$ residue was positively correlated with the activity of PP1; the interactions for $\mathrm{Mn}^{2+}$ and $\mathrm{Asn}_{124} / \mathrm{His}_{248}$ residues were negatively correlated with the activity of PP1. For the second $\mathrm{Mn}^{2+}$ ion (Figure 7(b)), its ion bond with Asp $_{64}$ remained constant but its ion bond with $\mathrm{His}_{66}$ increased when MCLR/MCRR irreversibly bind to PP1. However, due to the decreased ion bond between $\mathrm{Mn}^{2+}$ and $\mathrm{Asp}_{92}$, the total ion bonds showed downtrend. When GSH was introduced, the ion bond between $\mathrm{Mn}^{2+}$ and $\mathrm{Asp}_{64}$ was totally destroy; the ion bond between $\mathrm{Mn}^{2+}$ and $\mathrm{His}_{66}$ was weakened. Though the interaction between $\mathrm{Mn}^{2+}$ and $\mathrm{Asp}_{92}$ was dramatically enhanced, the total ion bonds were still weakened. Accordingly, the interaction for $\mathrm{Mn}^{2+}$ and $\mathrm{Asp}_{92}$ residue was positively correlated with the activity of PP1; the interactions for $\mathrm{Mn}^{2+}$ and $\mathrm{Asp}_{64} / \mathrm{His}_{66}$ residues were negatively correlated with the activity of PP1. The toxicity of MCs to PP1 also could be controlled by enhancing or reducing the specific ion bonds between $\mathrm{Mn}^{2+}$ and related sites. 

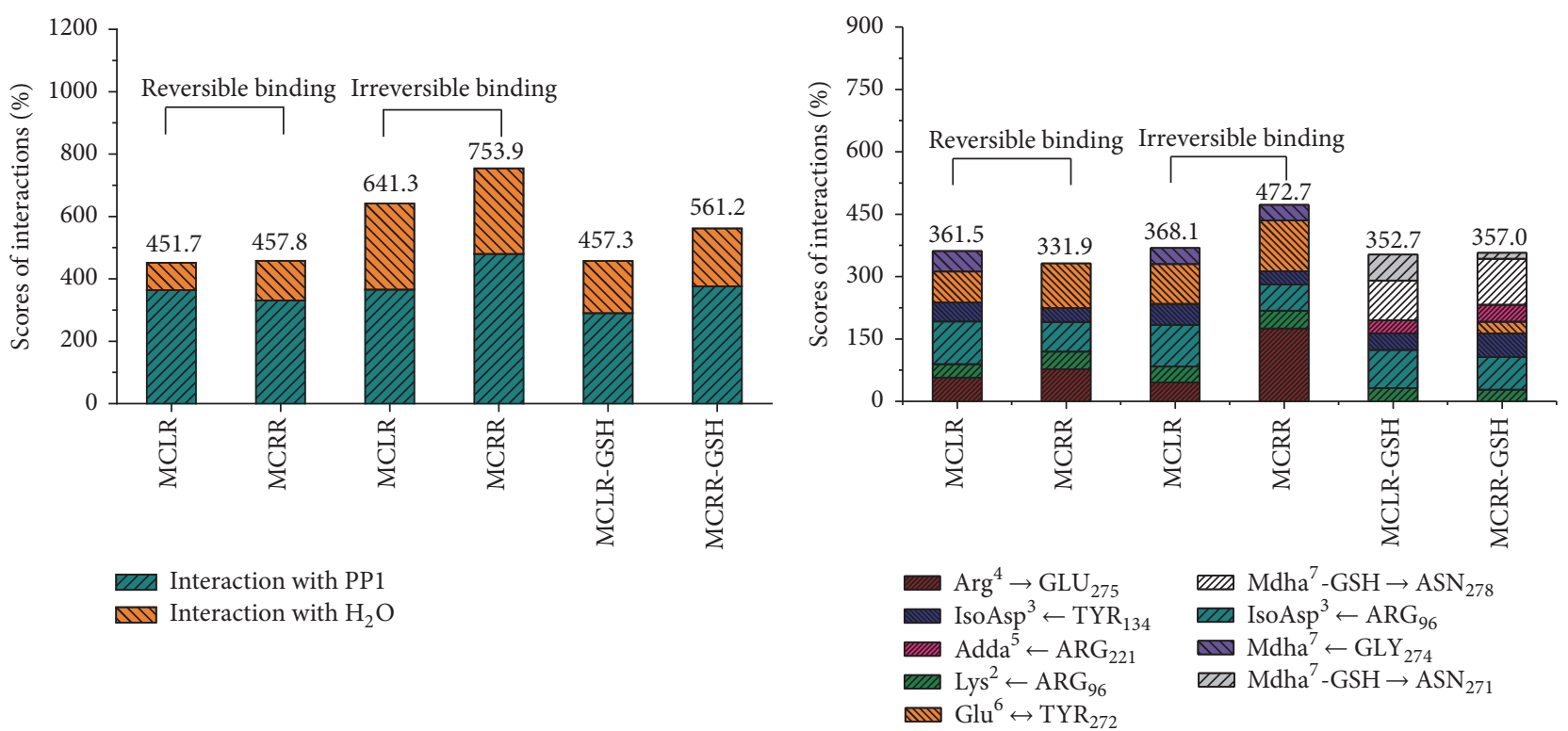

(a)

(b)

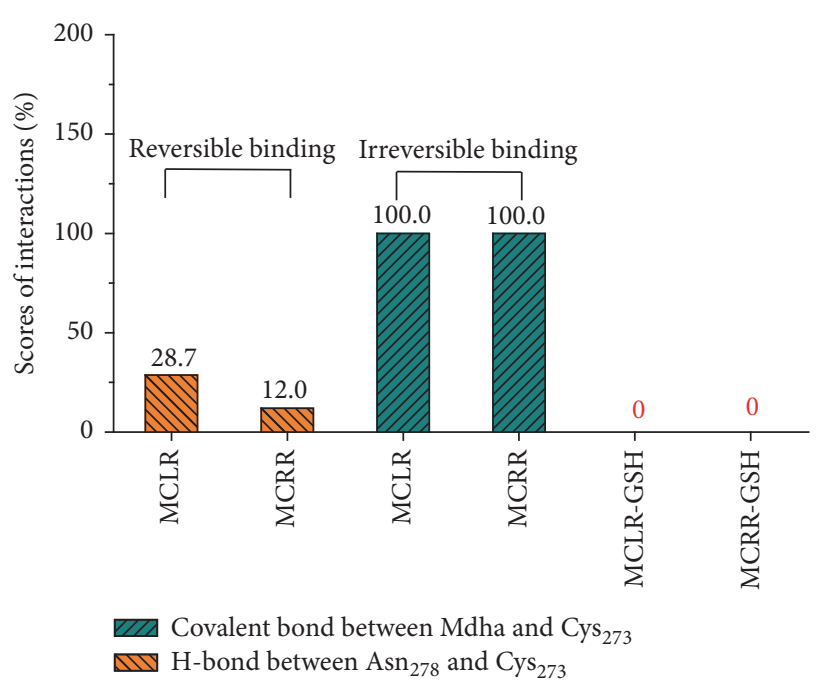

(c)

FIGURE 6: Scores for the total hydrogen bonds (a), the hydrogen bonds between primary interaction sites (b), and the specific covalent bonds between Mdha and $\mathrm{Cys}_{273}$ (c) of target complexes. Conditions: the total hydrogen bonds include the hydrogen bonds between MCs/MC-GSHs and $\mathrm{H}_{2} \mathrm{O}, \mathrm{MCs} / \mathrm{MC}-\mathrm{GSH}$, and PP1 ( $\mathrm{Arg}^{4}-\mathrm{Glu}_{275}$, $\mathrm{Lys}^{2}-\mathrm{Arg}_{96}$, IsoAsp ${ }^{3}-\mathrm{Arg}_{96}$, etc.).

\section{Conclusions}

Aiming at clarifying the detoxification mechanism of GSH conjugation pathway for the toxicity of MCs target to PPs, tow typical GSH conjugation products (MCLR-GSH/MCRRGSH) were prepared, separated, and purified. According to PP1 inhibition experiment, MCLR-GSH/MCRR-GSH showed evident control effect on the toxicity of MCs. Based on molecular simulation, the specific regulation mechanism of GSH conjugation pathway was clarified: data for combination area ascertained that the toxicity of MCs was controlled by enhancing the covalent binding of $\mathrm{Mdha}^{7} / \mathrm{Mdha}^{7}-\mathrm{GSH}$ to PP1, the hydrophobic interaction between $\mathrm{Adda}^{5}$ with PP1; data for combination energy showed the extra decreased trends for MC-GSHs with PP1 merely attributed to the weakened surface energy; data also showed the toxicity of MCs was controlled by enhancing the hydrogen bonds (especially for interaction sites $\mathrm{Lys}^{2}-\mathrm{Arg}_{96}$ and $\mathrm{Glu}^{6}-\mathrm{Tyr}_{272}$ ) and the covalent combination (between Mdha ${ }^{7}$ and $\mathrm{Cys}_{273}$ ). Specific investigation on the interactions involving $\mathrm{Mn}^{2+}$ ions in catalytic center also showed GSH conjugation promoted their interactions with $\mathrm{His}_{173} / \mathrm{Asp}_{92}$ residues but weakened the interactions with $\mathrm{Asn}_{124} / \mathrm{His}_{248} / \mathrm{Asp}_{64} / \mathrm{His}_{66}$ residues.

\section{Competing Interests}

The authors declare that they have no competing interests. 


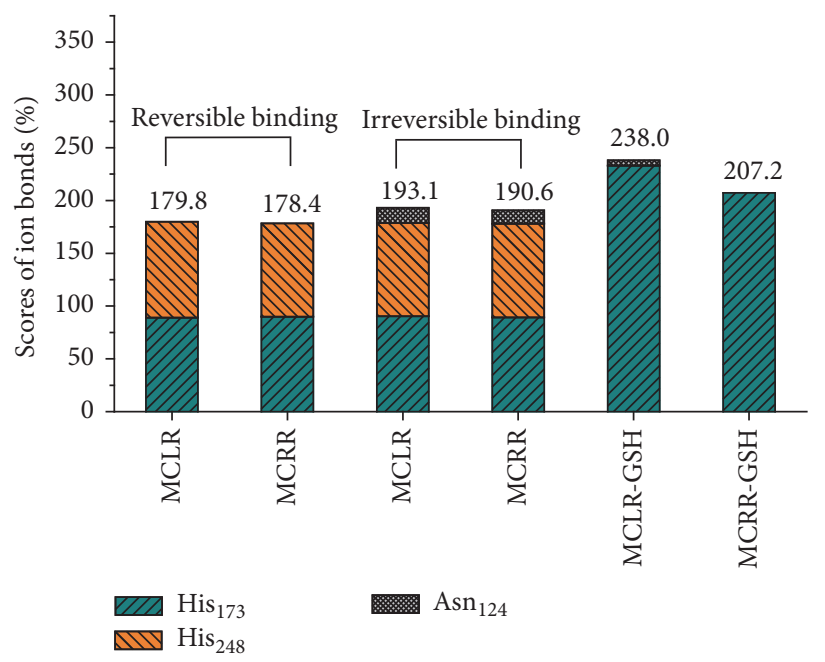

(a)

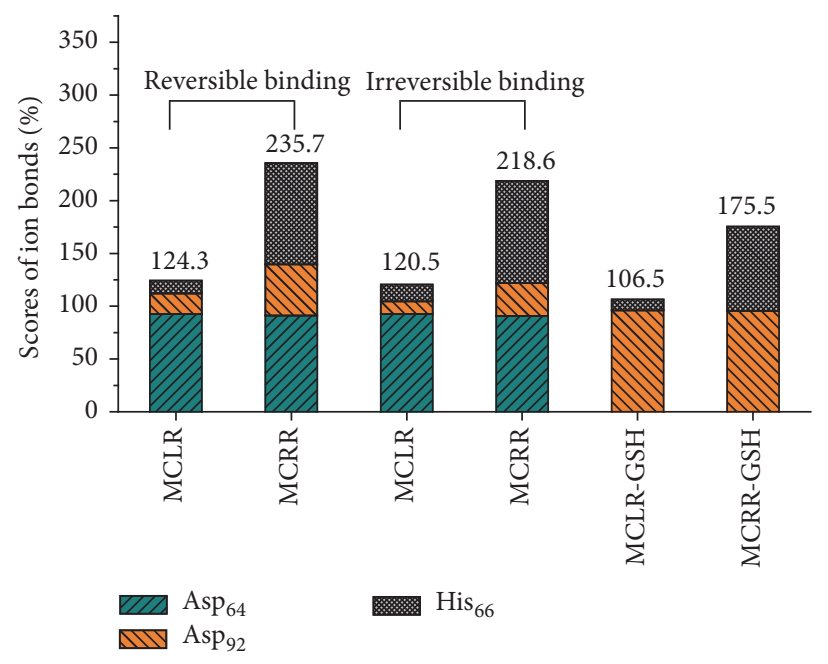

(b)

FIGURE 7: Scores for the major ion bonds involved in the interactions with $\mathrm{Mn}^{2+}$ ions in catalytic center of target complexes.

\section{Acknowledgments}

This work is supported by National Natural Science Foundation of China (21207082, 21577083, and 41606125) and Promotive Research Fund for young and middle-aged scientists of Shandong Province (BS2013HZ023).

\section{References}

[1] T. P. J. Kull, P. H. Backlund, K. M. Karlsson, and J. A. O. Meriluoto, "Oxidation of the cyanobacterial hepatotoxin microcystinLR by chlorine dioxide: reaction kinetics, characterization, and toxicity of reaction products," Environmental Science \& Technology, vol. 38, no. 22, pp. 6025-6031, 2004.

[2] D. Zhang, P. Xie, Y. Liu, and T. Qiu, “Transfer, distribution and bioaccumulation of microcystins in the aquatic food web in Lake Taihu, China, with potential risks to human health," Science of the Total Environment, vol. 407, no. 7, pp. 2191-2199, 2009.

[3] L. Chen, J. Chen, X. Z. Zhang, and P. Xie, "A review of reproductive toxicity of microcystins," Journal of Hazardous Materials, vol. 301, pp. 381-399, 2016.

[4] D. R. De Figueiredo, U. M. Azeiteiro, S. M. Esteves, F. J. M. Gonçalves, and M. J. Pereira, "Microcystin-producing blooms-a serious global public health issue," Ecotoxicology and Environmental Safety, vol. 59, no. 2, pp. 151-163, 2004.

[5] C. W. Diehnelt, S. M. Peterman, and W. L. Budde, "Liquid chromatography e tandem mass spectrometry and accurate $\mathrm{m} / \mathrm{z}$ measurements of cyclic peptide cyanobacteria toxins," $\operatorname{Tr} A C$ Trends in Analytical Chemistry, vol. 24, no. 7, pp. 622-634, 2005.

[6] M. G. Antoniou, A. A. de la Cruz, and D. D. Dionysiou, "Cyanotoxins: new generation of water contaminants," Journal of Environmental Engineering, vol. 131, no. 9, pp. 1239-1243, 2005.

[7] L. Pearson, T. Mihali, M. Moffitt, R. Kellmann, and B. Neilan, "On the chemistry, toxicology and genetics of the cyanobacterial toxins, microcystin, nodularin, saxitoxin and cylindrospermopsin," Marine Drugs, vol. 8, no. 5, pp. 1650-1680, 2010.

[8] B. M. Gulledge, J. B. Aggen, H. Eng, K. Sweimeh, and A. R. Chamberlin, "Microcystin analogues comprised only of Adda and a single additional amino acid retain moderate activity as PP1/PP2A inhibitors," Bioorganic \& Medicinal Chemistry Letters, vol. 13, no. 17, pp. 2907-2911, 2003.

[9] B. Žegura, B. Sedmak, and M. Filipič, "Microcystin-LR induces oxidative DNA damage in human hepatoma cell line HepG2," Toxicon, vol. 41, no. 1, pp. 41-48, 2003.

[10] M. Craig, H. A. Luu, T. L. McCready, C. F. B. Holmes, D. Williams, and R. J. Andersen, "Molecular mechanisms underlying the interaction of motuporin and microcystins with type-1 and type-2A protein phosphatases," Biochemistry and Cell Biology, vol. 74, no. 4, pp. 569-578, 1996.

[11] J. T. Maynes, K. S. Bateman, M. M. Cherney et al., "Crystal structure of the tumor-promoter okadaic acid bound to protein phosphatase-1," The Journal of Biological Chemistry, vol. 276, no. 47, pp. 44078-44082, 2001.

[12] M. Carneiro, B. Reis, J. Azevedo et al., "Glutathione transferases responses induced by microcystin-LR in the gills and hepatopancreas of the clam Venerupis philippinarum," Toxins, vol. 7, no. 6, pp. 2096-2120, 2015.

[13] B. Reis, M. Carneiro, J. Machado, J. Azevedo, V. Vasconcelos, and J. C. Martins, "Transcriptional responses of glutathione transferase genes in Ruditapes philippinarum exposed to microcystin-LR," International Journal of Molecular Sciences, vol. 16, no. 4, pp. 8397-8414, 2015.

[14] F. Kondo, Y. Ikai, H. Oka et al., "Formation, characterization, and toxicity of the glutathione and cysteine conjugates of toxic heptapeptide microcystins," Chemical Research in Toxicology, vol. 5, no. 5, pp. 591-596, 1992.

[15] R. W. MacKintosh, K. N. Dalby, D. G. Campbell, P. T. W. Cohen, P. Cohen, and C. MacKintosh, "The cyanobacterial toxin microcystin binds covalently to cysteine- 273 on protein phosphatase 1," FEBS Letters, vol. 371, no. 3, pp. 236-240, 1995.

[16] J. Chen, D. W. Zhang, P. Xie, Q. Wang, and Z. M. Ma, "Simultaneous determination of microcystin contaminations in various vertebrates (fish, turtle, duck and water bird) from a large eutrophic Chinese lake, Lake Taihu, with toxic Microcystis blooms," Science of the Total Environment, vol. 407, no. 10, pp. 3317-3322, 2009.

[17] S. Pflugmacher, C. Wiegand, A. Oberemm et al., "Identification of an enzymatically formed glutathione conjugate of the 
cyanobacterial hepatotoxin microcystin-LR: the first step of detoxication," Biochimica et Biophysica Acta (BBA)-General Subjects, vol. 1425, no. 3, pp. 527-533, 1998.

[18] J. S. Metcalf, K. A. Beattie, S. Pflugmacher, and G. A. Codd, "Immuno-crossreactivity and toxicity assessment of conjugation products of the cyanobacterial toxin, microcystin-LR," FEMS Microbiology Letters, vol. 189, no. 2, pp. 155-158, 2000.

[19] T. Heresztyn and B. C. Nicholson, "Determination of cyanobacterial hepatotoxins directly in water using a protein phosphatase inhibition assay," Water Research, vol. 35, no. 13, pp. 3049-3056, 2001.

[20] W. Zong, X. Wang, C. Yang, Y. Du, W. Sun, and Z. Xu, "Novel biomarker pipeline to probe the oxidation sites and oxidation degrees of hemoglobin in bovine erythrocytes exposed to oxidative stress," Biomedical Chromatography, pp. 810-817, 2015.

[21] X. Guo, L. Chen, J. Chen et al., "Quantitatively evaluating detoxification of the hepatotoxic microcystin-LR through the glutathione (GSH) pathway in SD rats," Environmental Science and Pollution Research, vol. 22, no. 23, pp. 19273-19284, 2015.

[22] W. S. Zong, F. Sun, and X. J. Sun, "Oxidation by-products formation of microcystin-LR exposed to $\mathrm{UV} / \mathrm{H}_{2} \mathrm{O}_{2}$ : toward the generative mechanism and biological toxicity," Water Research, vol. 47, no. 9, pp. 3211-3219, 2013.

[23] W. Zong, F. Sun, and X. Sun, "Evaluation on the generative mechanism and biological toxicity of microcystin-LR disinfection by-products formed by chlorination," Journal of Hazardous Materials, vol. 252-253, pp. 293-299, 2013.

[24] C. F. B. Holmes, J. T. Maynes, K. R. Perreault, J. F. Dawson, and M. N. G. James, "Molecular enzymology underlying regulation of protein phosphatase-1 by natural toxins," Current Medicinal Chemistry, vol. 9, no. 22, pp. 1981-1989, 2002.

[25] A. Campos and V. Vasconcelos, "Molecular mechanisms of microcystin toxicity in animal cells," International Journal of Molecular Sciences, vol. 11, no. 1, pp. 268-287, 2010. 

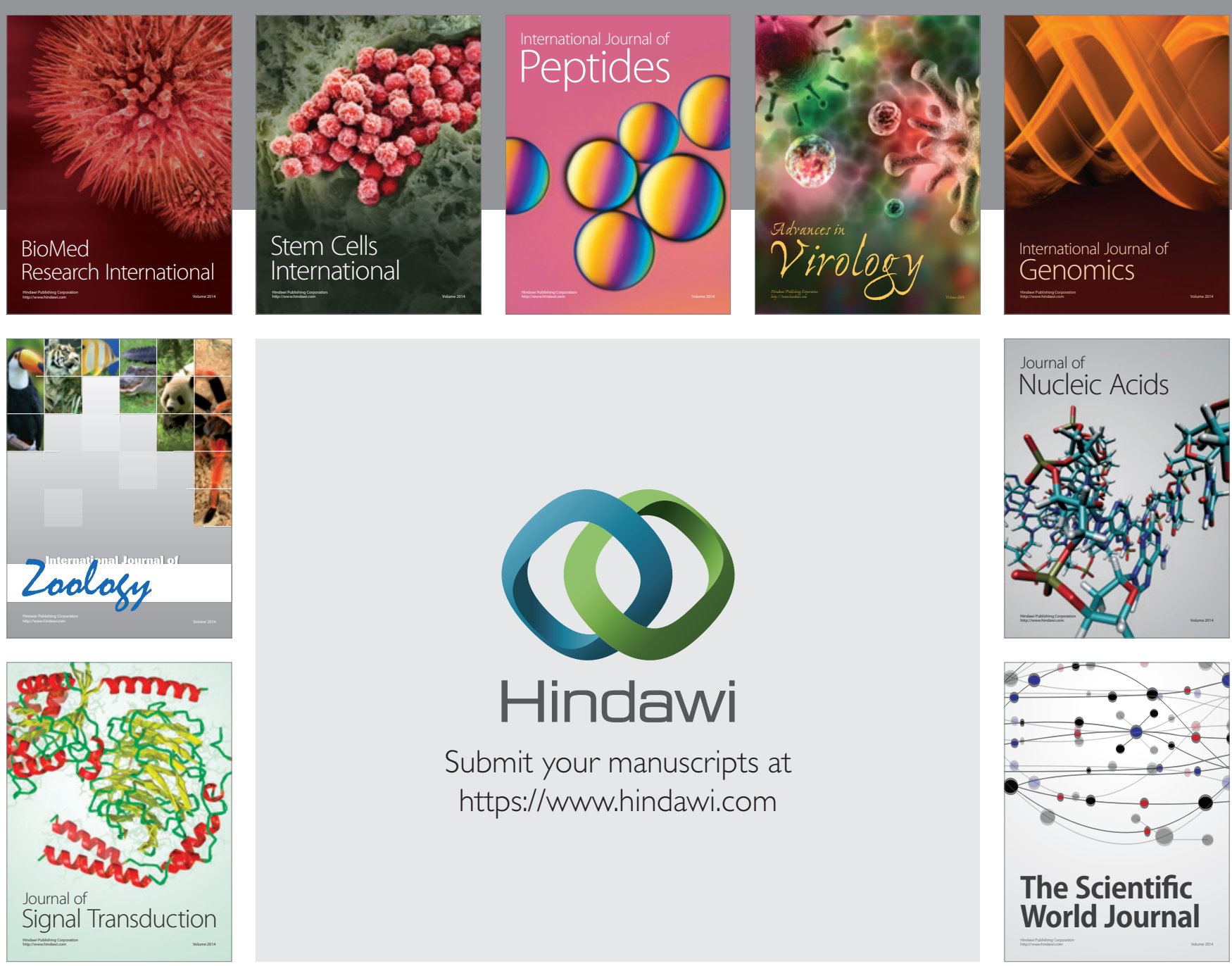

Submit your manuscripts at

https://www.hindawi.com
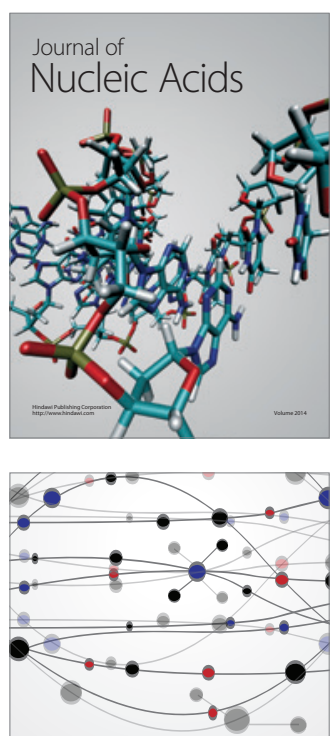

The Scientific World Journal
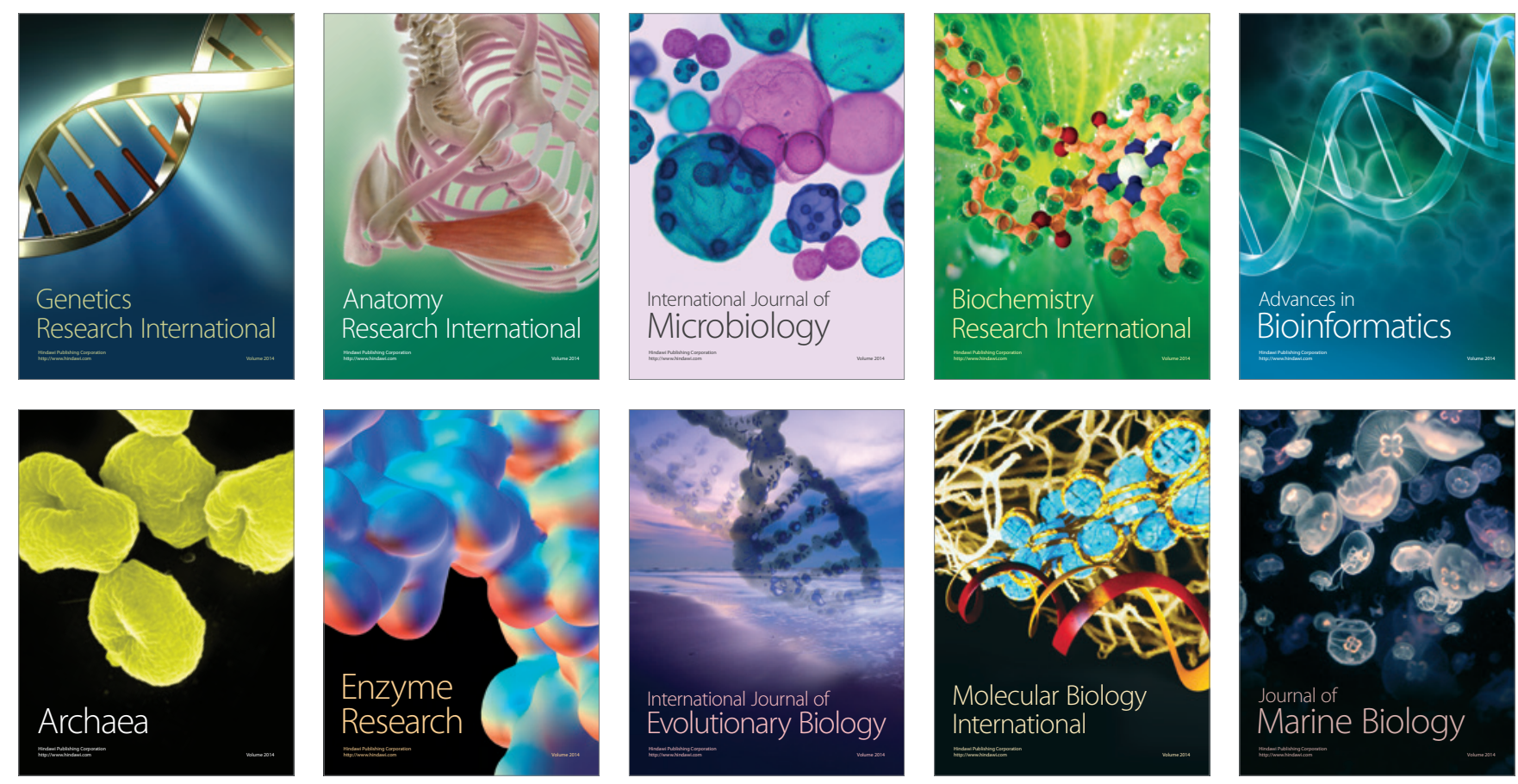